\title{
Eye Blink Classification for Assisting Disability to Communicate Using Bagging and Boosting
}

\author{
Luthfi Ardi ${ }^{1}$, Noor Akhmad Setiawan ${ }^{2}$, Sunu Wibirama ${ }^{3}$
}

\begin{abstract}
Disability is a physical or mental impairment. People with disability have more barriers to do certain activity than those without disability. Moreover, several conditions make them having difficulty to communicate with other people. Currently, researchers have helped people with disabilities by developing brain-computer interface (BCI) technology, which uses artifact on electroencephalograph (EEG) as a communication tool using blinks. Research on eye blinks has only focused on the threshold and peak amplitude, while the difference in how many blinks can be detected using peak amplitude has not been the focus yet. This study used primary data taken using a Muse headband on 15 subjects. This data was used as a dataset classified using bagging (random forest) and boosting (XGBoost) methods with python; $80 \%$ of the data was allocated for learning and $20 \%$ was for testing. The classified data was divided into ten times of testing, which were then averaged. The number of eye blinks' classification results showed that the accuracy value using random forest was $77.55 \%$, and the accuracy result with the XGBoost method was $90.39 \%$. The result suggests that the experimental model is successful and can be used as a reference for making applications that help people to communicate by differentiating the number of eye blinks. This research focused on developing the number of eye blinks. However, in this study, only three blinking were used so that further research could increase these number.
\end{abstract}

Keywords-Brain-Computer Interface (BCI), Electroencephalograph (EEG), Eye Blinks, Disability.

\section{INTRODUCTION}

Disability may include physical or mental inability or impairment. Even though there is no limitation for people with disability to carrying out activities, there are several conditions causing them to have communication barriers and make it difficult for them to communicate with others, such as in patients with amyotrophic lateral sclerosis (ALS) [1], lockedin syndrome, and other neurological disorders [2]. Over the last few years, some researchers have used brain signals with an electroencephalograph (EEG) to assist people with disabilities communicate. Brain-computer interface (BCI) is an external device that can be employed directly to communicate with the brain through certain devices that produce electronic wave signals from the brain [3] recorded by electrical components, called electrodes. This device is attached to certain positions on the scalp and is connected to the EEG devices. In addition, it can be identified as an instruction to be carried out by the organs.

1,2,3 Department of Electrical and Information Engineering, Faculty of Engineering, Universitas Gadjah Mada, Jln. Grafika No. 2, Kampus UGM, Yogyakarta, 55281, INDONESIA (phone: 0274552305; email: $\quad{ }_{1}^{1}$ luthfiardi@mail.ugm.ac.id, ${ }^{2}$ noorwewe@.ugm.ac.id. ${ }^{3}$ sunuwibrama@mail.ugm.ac.id)
An EEG is a recording of electrical activity along the scalp produced by firing neurons in the brain. It refers to a record of the brain's spontaneous electrical activity over a short period, usually for 20-40 minutes, in which several electrodes are placed on the scalp. In the EEG signal recording along the scalp, there are brain rhythms and artifacts. Brain rhythm is normal brain rhythms or main signals generated from the EEG recording. Artifacts are signal whose EEG recording activity contain many other signal disturbances such as ocular (eye), muscle, and heart. The stereoelectroencephalography signal records all brain activities so that there are several biases, one of which is an eye blink signal. Eye blink signal is a bias that researchers did not usually use, but today, the eye blink signal development obtained through the EEG recording process has been utilized by researchers. Until now, research on eye blink signals have only focused on the threshold at a certain amplitude, but there has not been much research related to the difference in eye blink signals based on the number of eyes blinks variation formulated on single blinking, double blinking, and triple blinking. In this study, the author developed the BCIbased experimental model of detecting and classifying the blink signal based on the number of blinks defined as single blinking, double blinking, and triple blinking.

\section{RELATED WORK}

EEG is an electrical activity recorded by placing multiple electrodes on the scalp. Today, EEG is widely used in neuroscience, cognitive sciences, cognitive psychology, neurolinguistics, and psychological study. In the recorded EEG signal, the signal can be divided into two, namely the brain rhythm and artifact.

Brain rhythm is a normal brain rhythm or the main signal resulted from EEG recording. Artifacts are signals of EEG's record activity which discovers other signal disturbance like ocular (eye), muscle, and heart. Some clinical conditions can be characterized by this brain rhythm.

The study about BCI and EEG has been extensively developed by the researchers. They start it by recording the brain activity produced by one of the EEG tools. However, researchers encounter constraints as there are other activities or instructions such as eye blink, muscles, and heart artifacts. Researchers have tried to dispose of artifacts found in the EEG record activity. Currently, researchers have used many of these artifacts to make them usable, as studied by [4]-[6] which were still focused on people who have difficulties to communicate, such as in people with disabilities and ALS disorder. Another research studied control DC motors on wheelchairs with eye blink signal based on LabVIEW [7]. This research used EOG signal produced when the eyes are blinking. Meanwhile, 


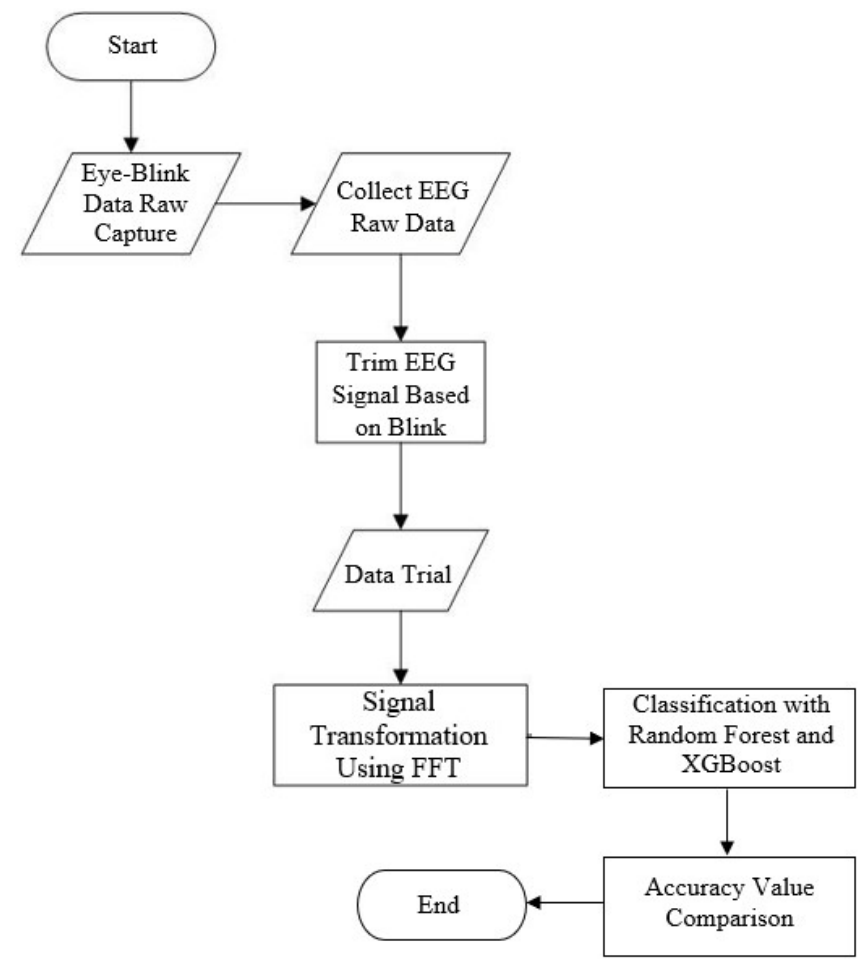

Fig. 1 System design flow.

research conducted by [8] examined lie detector by utilizing eye blink signal detection on EEG signal.

There are several research methods models that have been developed and used for blink detection to be used as a communication medium. Some of these methods utilized intentional detection of intentional blinking. Research [5] proposed detecting intentional and unintentional blink signals using NeuroSky EEG headset, the electrodes were placed on the FP1, the EEG data retrieval with intentional blinking was assisted by a virtual keyboard and one blink retrieval. Researchers [4] conducted a blink study which could select text options on a computer screen using an emotive tool with the number of blinks was one time, by calculating the covariance matrix and normalization to obtain the blink signal threshold; the electrodes used were AF3, AF4, F7, and F8. Research [6] used a blink signal to create a control design for patient room facilities using eye blink by alternately displayed four images on a computer screen. After selecting the right image, the patient would blink once with the electrode on FP1. With attention-level decision-making method of blinking obtained, this study used the NeuroSky tool. Research [9] used eye blink to communicate by stringing words and media to convey meaning with spoken words or body language. This study used the NeuroSky Mindware tool. Research [10] used eye blink to fold and open an electric bed in a hospital by using a virtual keyboard LabVIEW platform and selecting blocks or characters on a virtual keyboard, by determining the kurtosis coefficient values and the amplitude of the blink characteristics by using the Biopac MP36 tool at the electrode which were pasted on the FP1 and FP3. Research [11] used blinks to control the light activation system at home by placing electrodes on the frontal, earlobe, and occipital areas. This research subjects were
20 people who were instructed to close and open their eyes thrice every two and three seconds, respectively.

However, the research mentioned still employs auxiliary media such as images on a computer screen that are replaced within a certain period to a virtual keyboard where the patient is required to blink according to the letter. Using duration to discover the communication's purpose requires longer time and is very difficult to understand as well as to carry out. The blink detection carried out in the previous research used a single threshold for one blink of an eye. Therefore, the application of the different number of blinks in which each number conveys a meaning is very likely to be a solution, for example blinking once means want to drink, blinking twice means to eat, and blinking thrice means sick. The difference in the number of blinks is very concise to communicate directly; at one, two, and three blinks, the threshold shown is the difference pattern. Thus, this study used one to three eye blinks as a signal to communicate.

\section{METHODS}

This section presents the methods used in this study.

\section{A. Participant}

Fifteen male students (mean age: 23 years old) from Universitas Gadjah Mada participated in the experiment using the portable Muse EEG system. All participants had normal or corrected-to-normal vision with no neurological impairments.

\section{B. System Design}

This study proposed a BCI system designed for communicating using the number of eye blinks detected from the EEG signal.

This research started by taking the blinking data, then collected the raw data. Afterward, the collected raw data was cut and made into a dataset which then transformed using fast Fourier transform (FFT). The following step was classifying the dataset using random forest and XGboost, then compared the results that have been achieved.

The number of blinks was defined as single blink, double blinks, and triple blinks. The number of samples used in this study was 15 male students who were set with a Muse headband on the head.

Before test was conducted, students were asked to enter a room in turn, sat back, and relax. The participants were given instructions prior to wear the Muse headband. The research time was 15 seconds and a stopwatch was placed in front of them. At the fifth seconds, they must blink once; at the tenth seconds, they must blink twice; and at the fifteenth seconds, they must blink thrice. This process is illustrated in Fig. 1.

\section{EEG Acquisitions}

This study used a portable brain signal sensor called Muse manufactured by the InteraXon Company based in Toronto, Ontario, Canada [12]. This company was developed in 2007 by Ariel Garten, Trevor Coleman, Chris Aimone, and Steve Mann [13]. Muse is a wearable brain-sensing headband. This portable headset measures brain activity via four EEG sensors. The researchers obtain EEG signals from the subject's brain by 


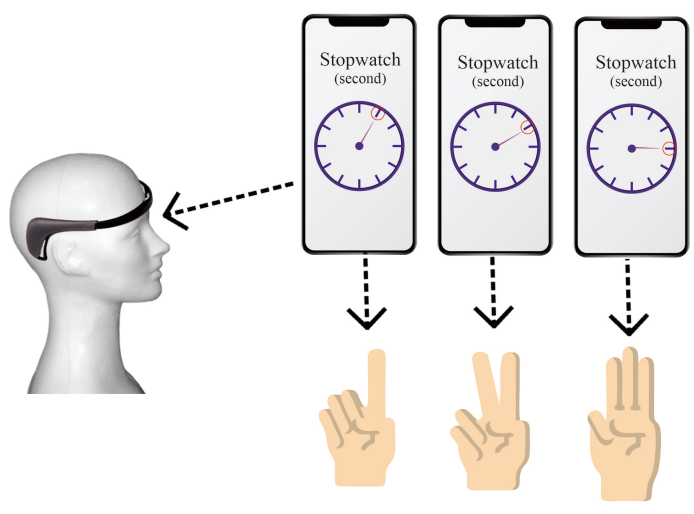

Fig. 2 Design of how to capture EEG signal using eye blinks.

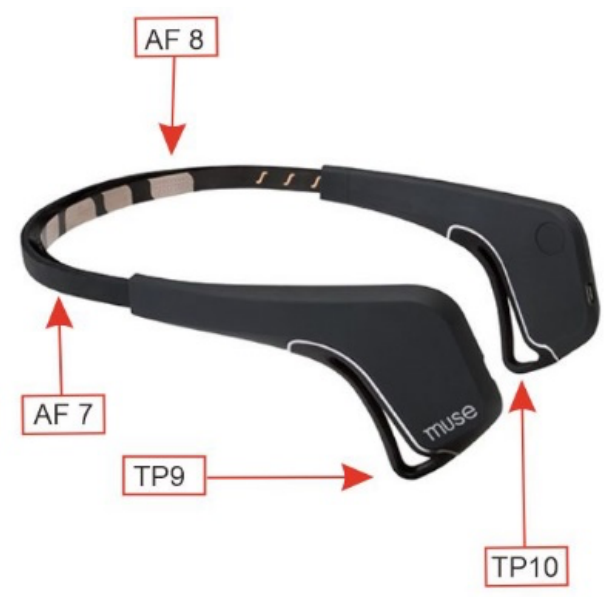

Fig. 3 Muse headband sensor.

placing the electrodes on the frontal part [13] as shown in Fig. 2. This EEG signal will be obtained by placing the headset on the frontal; the headset is connected to Bluetooth and to an application called Muse App on the smartphone. Afterward, the EEG signal can be sent to Dropbox.

As in Fig. 3, Muse headband has four sensors, namely AF7 sensor placed on the left side of the eyebrow, AF8 sensor placed on the right side of the eyebrow, TP9 sensor placed on the back of the left ear, and TP10 sensor placed on the back of the right ear. The followings are Muse headband specifications.

- Four Channels, two on the forehead, two behind the ears.

- Communicating via Bluetooth.

- Generating bipolar readings using AFz as reference for AF7, AF8, TP9, TP10.

- $3 \mathrm{CMS} / \mathrm{DRL}$ electrical reference electrodes Montage 7 Sendoe which allows estimation of hemispheric asymmetry (with emphasis on the pre-frontal lobe) and thereby facilitates discrimination of brain conditions depending on asymmetry, such as emotional valence.

- Create the output from $220 \mathrm{~Hz}$ to $500 \mathrm{~Hz}$, with $2 \mathrm{uV}$ (RMS) noise.

- The input range of the AC pair signal (low cutoff at $1 \mathrm{~Hz}$ ) is $2 \mathrm{mV}$ - $\mathrm{p}$.

According to 10-20 system, sensors recorded on the Muse headband are four channels, namely AF7, AF8, TP9, and TP10, as shown in Fig. 4

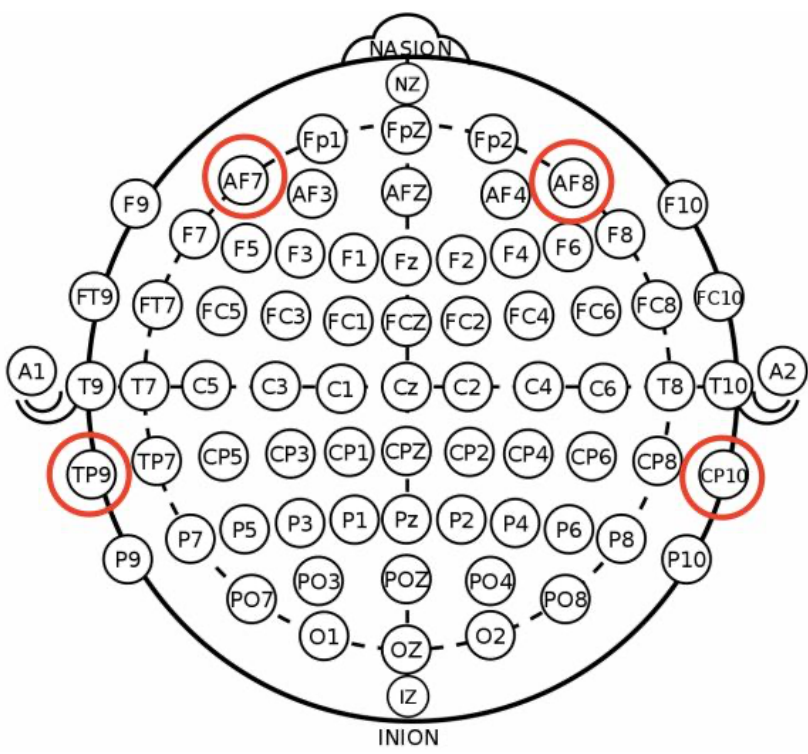

Fig. 4 EEG electrode placement.

\section{Data Processing}

The collected participants' brain signals were used as a dataset by cutting any data from any time containing a value of single blinking, double blinking, dan triple blinking so that the computer can learn the data.

The process of creating a dataset used the results of imported brain signal recording from Muse using EEGLAB on Matlab with the help of the extension "Import from Muse Monitor App. CSV File". Since the data from muse separates the delta, theta, alpha, beta, and gamma signals on each channel, then the signals were imported using extension imports from EEGLAB and the results of the data were made into one data for each channel.

After the signal data has been collected, the data from the EEGLAB was exported into a text file. The file that has been exported was then imported to Matlab and became the value on Matlab. In recording the EEG signal, there were four recorded channels, namely AF7, AF8, TP9, and TP10. Due to the highest signal value obtained on the recording was TP9, the signal taken was only at TP9.

The TP9 signal is the part of raw data that has single blinking, double blinking, and triple blinking values and it was separated from the data obtained. TP9 signal was then plotted using the participants' 250 frame rate to be the actual seconds, the signal from the TP9 channel plot can be seen in Fig. 5.

Fig. 5 shows the plot of the complete EEG data result. At the fifth second, the signal's peak is only one, indicating that there is only one blink. At the tenth second, two signal peaks indicates that there are two blinks. At the fifteenth second, three peak points indicating that there are three blinks.

The data were combined into one complete data subject, or there were three pieces of information on single blinking and double blinking, as seen on Fig. 5. The sampling for the learning process used machine learning methods; then, computer classification is more targeted, the data was cut by two seconds according to the information required. The two 


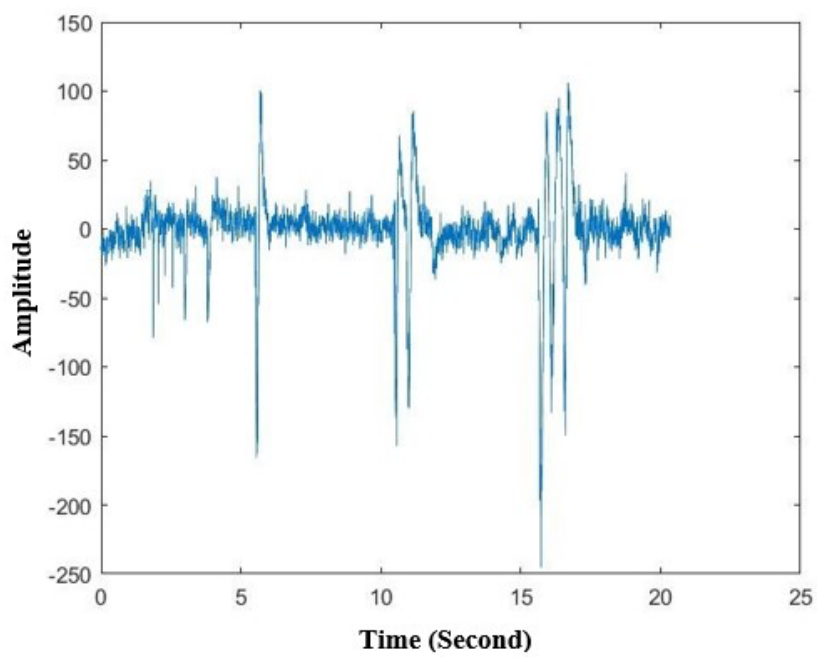

Fig. 5 Plot TP9 with fs 250.

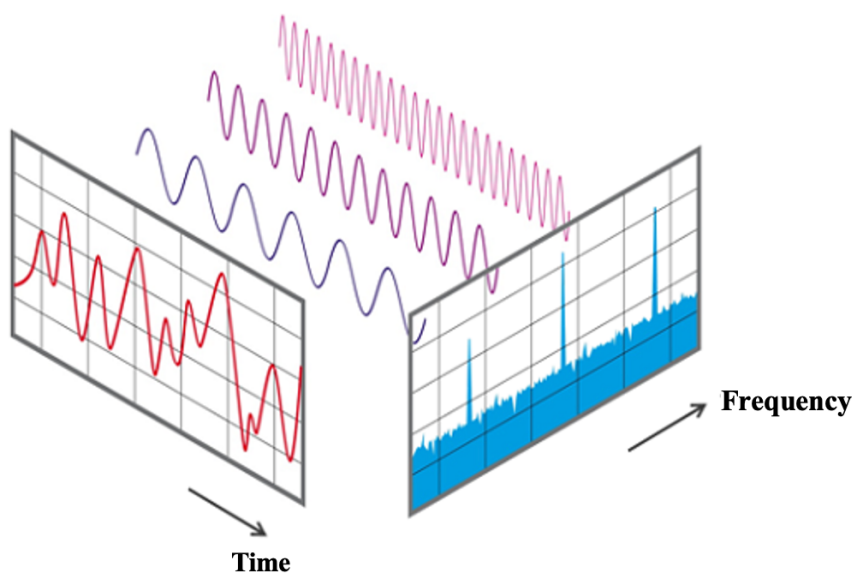

Fig. 6 FFT algorithm illustration.

seconds deduction was based on the average of single blinking, double blinking, and triple blinking in this study. The raw data was cut according to the blinks, then it was labeled according to the number of blinking. All participants data were cut into one dataset.

\section{E. Signal Transformation}

The created dataset, which was in the time domain, was transformed using the FFT method into a signal in the frequency domain, such as Fig. 6. In general, the EEG signal is highly complex and non-linear, so it would be better to use a non-linear model. In this study, FFT was utilized to extract the features of signal eye blinks.

Signals are usually measured in the time domain, but they require spectral specifications in the frequency domain for analysis. Signal synthesis is easier to carry out in the frequency domain since the quantity that most determines a signal is a frequency. Periodic signals can be analyzed using the Fourier series; as for non-periodic signals, it can be analyzed using discrete Fourier transform (DFT). However, the problem is that DFT is very complex, complicated, and requires a very large amount of computation. To overcome these problems, the FFT algorithm was used as it has only $N \log _{2} N$ of computations. By

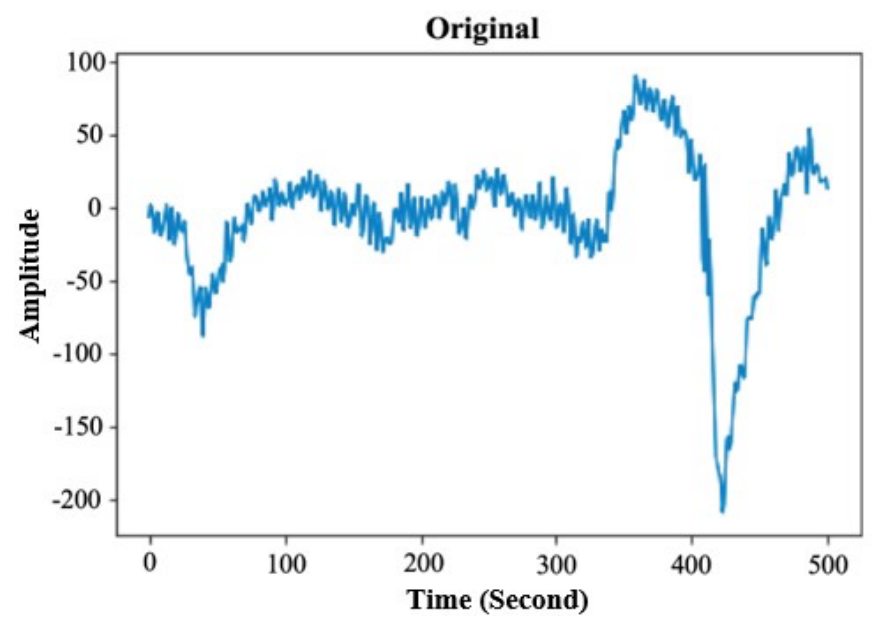

(a)

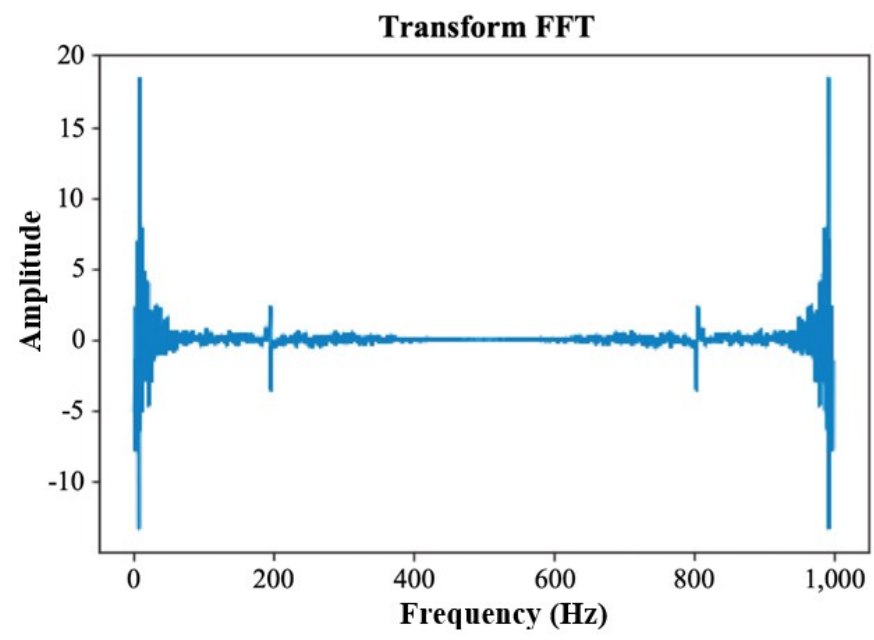

(b)

Fig. 7 Comparison of the original plot and in FFT single blinking: (a) before FFT and (b) after FFT.

using FFT, the frequency spectrum can be quickly generated, and the main complex signal's component spectrum can be easily recognized. Furthermore, signals can be properly processed by applying the threshold into the single signal's amplitude which can be produced without much changing the original signal.

Hence, the values of a given time-series data as a numeric sequence data were converted into a finite set of the frequency domain. Then, to deconstruct signals into segmented EEG signal sequences, FFT was used to convert a signal from its original, from time domain to a representation in the frequency domain signal [14]. Fig. 7 represents the form of a time-domain signal. Previous studies have shown that FFT is a promising tool for stationary signal processing, and the fastest method to get the result, and its more appropriate for sine waveforms such as in EEG signals [15].

Fig. 7 is an example of the recorded dataset results comparison from a single blinking FFT transform; Fig. 7(a) is the original single blinking plot and Fig. 7(b) is the single blinking FFT transform results. Fig. 8 is an example of the double blinking FFT transformed dataset comparison; Fig. 8(a) 


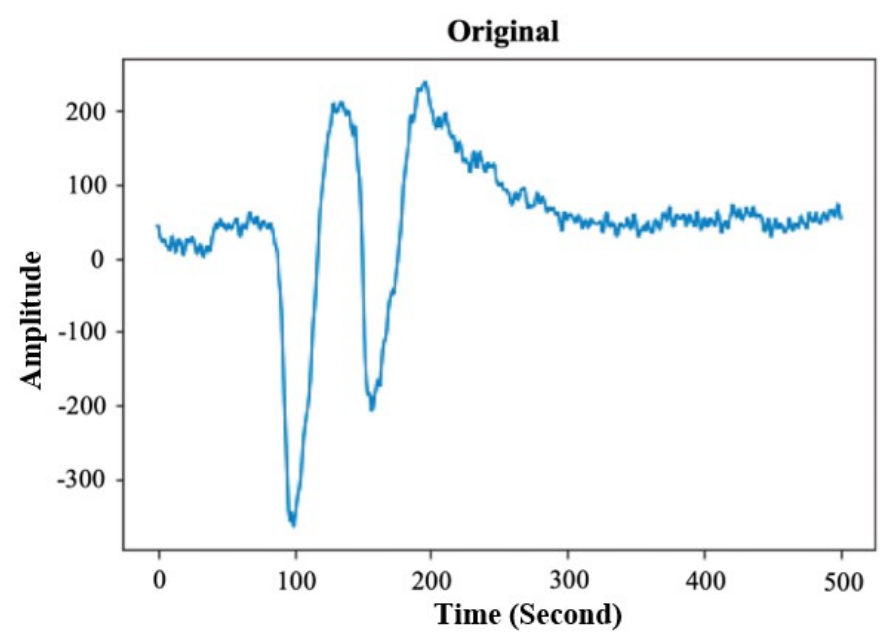

(a)

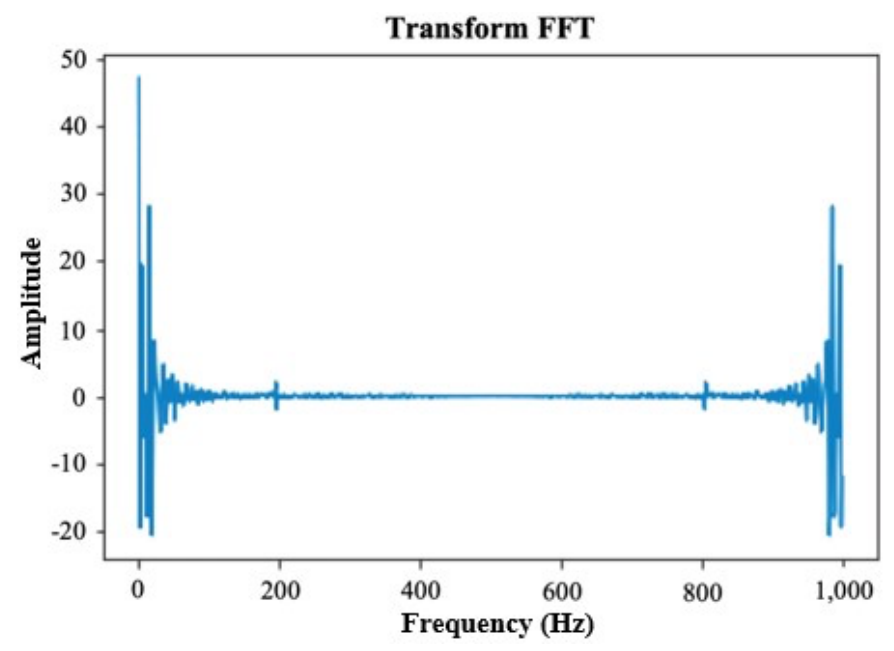

(b)

Fig. 8 Comparison of the original plot and after in FFT double blinking: (a) before FFT and (b) after FFT.

is the original double-blinking plot and Fig. 8(b) is the doubleblinking FFT transformation. Fig. 9 is an example of the recording for the triple blinking FFT transformed dataset comparison; Fig. 9(a) is the original triple blinking plot and Fig. 9(b) is the triple blinking FFT transform.

Looking directly at the original signal form in single, double, and triple blinking, the difference in the number of the blinking's peak is easy to notice. However, learning machine cannot maximally classify the original data. Thus, the original signal in the FFT and the shape of the signal should be changed to frequency.

\section{F. Signal Classification}

After the dataset was transformed into FFT, to get the study results, the dataset was classified using bagging (random forest) [16] and boosting (XGBoost) [17] in python, which was allocated $80 \%$ for learning and $20 \%$ for testing.

The random forest and XGBoost methods are some of the methods in the decision tree [18]. A decision tree or decisionmaking tree is a tree-like shaped flow chart which has a root

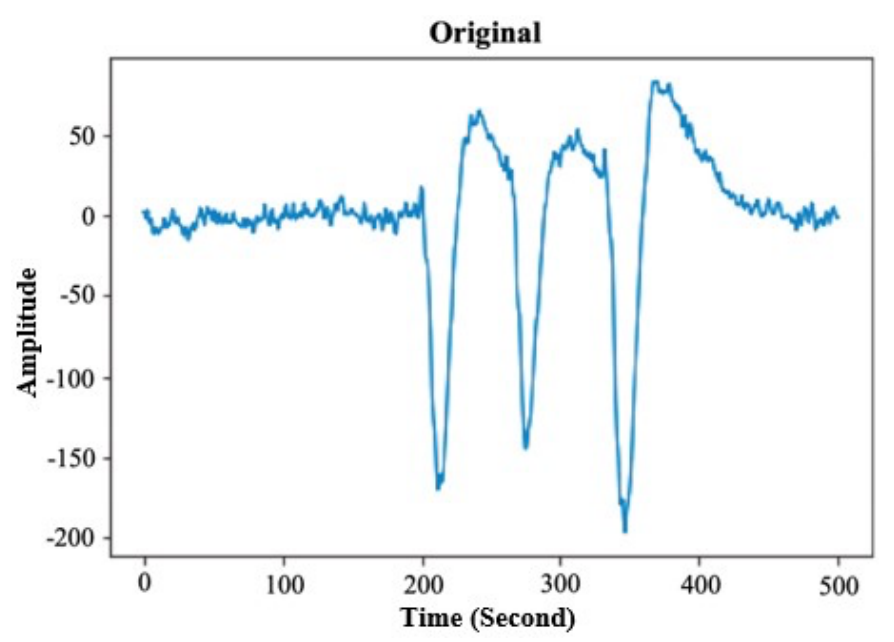

(a)

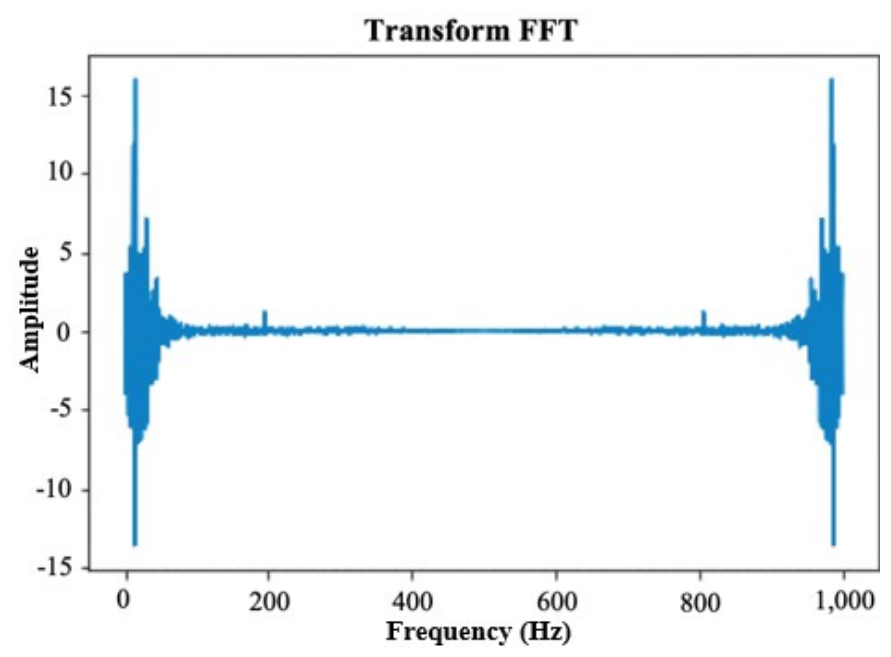

(b)

Fig. 9 Comparison of the original plot and after in FFT triple blinking: (a) before FFT and (b) after FFT.

node used to collect data. An inner node located at the root node contains questions about data, while a leaf node is used to solve problems and create the decision. The decision tree classifies a sample of data that is not yet known for its class into existing classes. The use of a decision tree is intended to avoid overfitting a data set while achieving maximum accuracy.

The random forest consists of trees combined into one model. Random forest depends on a random vector valued by the same distribution in all trees, each decision tree has the maximum depth. The random forest is a classifier consisting of a tree classifier $(h(x, \theta k), k=1, \ldots\}$ where $\theta k$ is a random vector distributed independently and each tree in a unit will choose the most popular class in the input $x$.

XGBoost is an algorithm used for classification and part of the tree family. The strengths of XGBoost include parallelism and hardware optimization. Data is stored in memory, called blocks, in a compressed column format, i.e., compressed sparse column (CSC). The algorithm can perform tree pruning to remove branches with low probability. The loss function model 
TABLE I

RESUlTS OF THE CLASSIFICATION OF RANDOM FOREST AND XGBOOST

\begin{tabular}{|c|c|c|}
\hline No. & Random Forest (\%) & XGBoost (\%) \\
\hline 1 & 82.14 & 100 \\
\hline 2 & 78.57 & 92.85 \\
\hline 3 & 74.07 & 77.77 \\
\hline 4 & 77.77 & 85.18 \\
\hline 5 & 81.48 & 81.48 \\
\hline 6 & 66.66 & 88.88 \\
\hline 7 & 92.59 & 88.88 \\
\hline 8 & 74.07 & 96.29 \\
\hline 9 & 85.18 & 96.29 \\
\hline 10 & 62.96 & 96.29 \\
\hline Average & 77.55 & 90.39 \\
\hline
\end{tabular}

has a term to measure the complexity of the model using regularization to streamline the learning process (reduce the possibility of overfitting).

The frame works well even with missing scores or many zeros with scatter awareness. XGBoost uses an algorithm called the "weighted quantile sketch algorithm", which allows the algorithm to focus on misclassified data. The goal of each new student is to learn how to classify the incorrect data after each iteration. This method sorts the data by quantity to find the right separation point. It is the objective of the parameter $\epsilon$, which is the split value $(\epsilon=0.1$; quantile $=[10 \%, 20 \%, \ldots, 90 \%])$.

XGBoost has a hyper-parameter (a parameter which is not studied by an estimator) which must be determined by parameter optimization. The process is simple, each parameter to be estimated is represented by a list of values, each combination is then tested by a model which metrics are compared to conclude the best combination. The Parameter searches the needs, and it is guided by metrics through silane validation.

\section{RESUlt}

This section presents the results of the research conducted. The results $t$ shown in this section begins with the dataset preparation process to the classification process to determine the significance of the result accuracy differences. As already explained that the dataset was obtained by taking primary data, which was taken by using Muse to retrieve the EEG signal from the participants' head. The participants were given instruction before wearing the Muse headband. The research time was 15 seconds and the stopwatch was placed in front of them. At the fifth seconds, they must blink once; at the tenth seconds, they must blink twice; and at the fifteenth seconds, they must blink thrice. The data were collected, then it was separated based on the electrode. In this study, the electrode used was TP9, the data raw on TP9 was then plotted according to time. Each raw data contained about 15-20 seconds of EEG recording and cut with a period of two seconds. The data that was cut was trimmed from the plot results showing the number of blinking, then the data on the subject that has been cut was labeled on each blink. The label was named according to the number of blinking, such as single-blinking is labeled 0 , double blinking labeled 1 , and triple-blinking labeled 2. Following data labeling, the data that has been cut and labeled was made into one dataset containing 15 subject data that has been cut and labeled. The dataset was then transformed to FFT. FFT was used to convert a signal from its original, from time domain to a representation in the frequency domain signal.

Afterward, dataset was classified in Python using random forest and XGboost methods, the results were split up to ten times testing. The results of the classification are shown in the Table I.

From the classification table for the data results above, the random forest method produces an average accuracy of $77.55 \%$, and the XGBoost method produces an average accuracy of $90.39 \%$. The results from the table have shown that the proposed method is successful, and the number of eye blinks was well-differentiated.

\section{CONCLUSION}

This contribution presented a development of a BCI system. Eye blinks were used to get single-blinking, double-blinking, and triple-blinking with peak threshold signal. Therefore, this method can be used as a reference to help people with disabilities to communicate. This research is limited to the development of application features. Additionally, this study only used triple blinking for classification. Thus, future work can increase the number of eye blinks by more than three blinks and improve the accuracy using another method to classify eye blinks.

\section{REFERENCES}

[1] P. Masrori and P. Van Damme, "Amyotrophic Lateral Sclerosis: A Clinical Review," European Journal of Neurology, Vol. 27, No. 10, pp. 1918-1929, Oct. 2020.

[2] F. Cincotti, D. Mattia, F. Aloise, S. Bufalari, G. Schalk, G. Oriolo, A. Cherubini, M.G. Marciani, and F. Babiloni, "Non-Invasive BrainComputer Interface System: Towards Its Application as Assistive Technology," Brain Research Bulletin, Vol. 75, No. 6, pp. 796-803, Apr. 2008.

[3] M.M. Fouad, K.M. Amin, N. El-Bendary, and A.E. Hassanien, "Brain Computer Interface: A Review," in Brain-Computer Interfaces, A.E. Hassanien and A.T. Azar, Eds. Cham, Switzerland: Springer International Publishing, 2015, pp. 3-30.

[4] S. Soman and B.K. Murthy, "Using Brain Computer Interface for Synthesized Speech Communication for the Physically Disabled," Procedia Computer Science, Vol. 46, pp. 292-298, Dec. 2015.

[5] K.S. Kabir, C.M.A. Rahman, A. Farayez, and M. Ferdous, "An Experimental Model of an Indigenous BCI Based System to Help Disabled People to Communicate," AIP Conference Proceedings, Vol. 1919, No. 1, 2017, pp. 1-7.

[6] I.A.E. Zaeni, A.P. Wibawa, and M. Ashar, "The Design of the Patient's Room Facilities Controller Using an Eye Blink," 2017 5th International Conference on Electrical, Electronics and Information Engineering (ICEEIE), 2017, pp. 88-91.

[7] W. Zhi-Hao, Hendrick, K. Yu-Fan, C. Chuan-Te, L. Shi-Hao, and J. GwoJia, "Controlling DC Motor Using Eye Blink Signals Based on LabVIEW," 2017 5th International Conference on Electrical, Electronics and Information Engineering (ICEEIE), 2017, pp. 61-65.

[8] J. Immanuel, A. Joshua, and S.T. George, "A Study on Using Blink Parameters from EEG Data for Lie Detection," 2018 International Conference on Computer Communication and Informatics (ICCCI), 2018, pp. 1-5.

[9] A.K. Singh, Y. Wang, J. King, C. Lin, and L. Ko "A Simple Communication System Based on Brain Computer Interface," 2015 
Conference on Technologies and Applications of Artificial Intelligence (TAAI), 2015, pp. 363-366.

[10] B. Chambayil, R. Singla, and R. Jha, "Virtual Keyboard BCI Using Eye Blinks in EEG," 2010 IEEE 6th International Conference on Wireless and Mobile Computing, Networking and Communications, 2010, pp. 466470.

[11] M.S.B.A. Rani and B.T.M. Wahidah, "Detection of Eye Blinks from EEG Signals for Home Lighting System Activation," 2009 6th International Symposium on Mechatronics and Its Applications, 2009, pp. 1-4.

[12] J.J. Bird, L.J. Manso, E.P. Ribeiro, A. Ekart, and D.R. Faria, "A Study on Mental State Classification Using EEG-Based Brain-Machine Interface," 2018 International Conference on Intelligent Systems (IS), 2018, pp. 795800.

[13] A. Przegalinska, L. Ciechanowski, M. Magnuski, and P. Gloor, "Muse Headband: Measuring Tool or a Collaborative Gadget?" in Collaborative Innovation Networks, Studies on Entrepreneurship, Structural Change and Industrial Dynamics, F. Grippa, J. Leitão, J. Gluesing, K. Riopelle, and P. Gloor, Eds., Cham, Switzerland: Springer International Publishing, 2018, pp. 93-101.
[14] M.K. Delimayanti, B. Purnama, N.G. Nguyen, M.R.Faisal, K.R. Mahmudah, F. Indriani, M. Kubo, and K. Satou, "Classification of Brainwaves for Sleep Stages by High-Dimensional FFT Features from EEG Signals," Applied Sciences, Vol. 10, No. 5, pp. 1-12, Mar. 2020.

[15] A.S. Al-Fahoum and A.A. Al-Fraihat, "Methods of EEG Signal Features Extraction Using Linear Analysis in Frequency and Time-Frequency Domains," ISRN Neuroscience, Vol. 2014, pp. 1-7, Feb. 2014.

[16] E. Goel and E. Abhilasha, "Random Forest: A Review," International Journal of Advanced Research in Computer Science and Software Engineering, Vol. 7, No. 1, pp. 251-257, 2017.

[17] T. Chen and C. Guestrin, "XGBoost: A Scalable Tree Boosting System," Proceedings of the 22nd ACM SIGKDD International Conference on Knowledge Discovery and Data Mining, 2016, pp. 785-794.

[18] S. Kabiraj, M. Raihan, N. Alvi, M. Afrin, L. Akter, S.A. Sohagi, and E. Podder, "Breast Cancer Risk Prediction Using XGBoost and Random Forest Algorithm," 2020 11th International Conference on Computing, Communication and Networking Technologies (ICCCNT), 2020, pp. 1-4. 